\title{
Comparação de modelos matemáticos para descrição das curvas de dessorção de sementes de milho-doce ${ }^{(1)}$
}

\author{
Eduardo Fontes Araújo(2), Paulo César Corrêa( ${ }^{(3)}$ e Roberto Ferreira da Silva(4)
}

\begin{abstract}
Resumo - Este trabalho teve como objetivo determinar as curvas de dessorção das sementes de milhodoce (Zea mays L.), cultivares Superdoce e Doce Cristal, e ajustar diferentes modelos matemáticos aos dados obtidos. As sementes das duas cultivares foram submetidas à dessorção em diversos níveis de temperatura $\left(30,40,50\right.$ e $\left.60^{\circ} \mathrm{C}\right)$, combinados com diferentes umidades relativas do ar $(30,40,50 \mathrm{e}$ $60 \%$ ), até atingirem a umidade de equilíbrio. Os seguintes modelos matemáticos foram ajustados por análise de regressão: Henderson-Thompson, Chung-Pfost, Copace, Sigma-Copace, Sabbah e Smith. As sementes das duas cultivares apresentaram umidades de equilíbrio higroscópico semelhantes. Os valores da variância explicada e do desvio-padrão, bem como a distribuição dos resíduos, das duas cultivares, indicam que as equações de Chung-Pfost, Sabbah e Smith foram as que melhor se ajustaram aos dados experimentais, com pequena superioridade da primeira.
\end{abstract}

Termos para indexação: Zea mays, atividade da água, teor de umidade, higroscopicidade.

\section{Comparison of mathematical models for description of desorption curves of sweet corn seeds}

Abstract - This work had the goal to determine the desorption curves of seeds of sweet corn cultivars Superdoce and Doce Cristal, and to adjust mathematical models to obtained data. Corn seeds were submitted to desorption under different temperature conditions $\left(30,40,50\right.$ and $\left.60^{\circ} \mathrm{C}\right)$, associated with different relative humidity $(30,40,50$ and $60 \%)$ until reaching the equilibrium humidity. The following models of regression analysis were adjusted to the experimental data: Henderson-Thompson, ChungPfost, Copace, Sigma-Copace, Sabbah and Smith. Seeds of both cultivars showed similar hygroscopic equilibrium. For both cultivars, the equations of Chung-Pfost, Sabbah and Smith, showed the best adjustments to the experimental data, with slight superiority of the first one.

Index terms: Zea mays, water activity, moisture content, hygroscopicity.

\section{Introdução}

O milho-doce, utilizado principalmente como milho verde, tanto in natura como para processamento, difere do milho comum, não por características taxonômicas, mas pelo alto teor de açúcares e baixo teor de amido, ambos resultantes da ação de

\footnotetext{
(1) Aceito para publicação em 22 de setembro de 2000. Parcialmente financiado pela Fundação de Amparo à Pesquisa do Estado de Minas Gerais (FAPEMIG).

${ }^{(2)}$ Universidade Federal de Viçosa (UFV), Dep. de Fitotecnia, CEP 36571-000 Viçosa, MG. E-mail: efaraujo@mail.ufv.br

(3)UFV, Dep. de Engenharia Agrícola.

E-mail: copace@mail.ufv.br

(4) Universidade Estadual do Norte-Fluminense (UENF), CEP 28015-620 Campos dos Goytacazes, RJ

E-mail: roberto@uenf.br
}

genes recessivos individuais ou associados em combinações duplas ou triplas. Além dessas combinações gênicas particulares, o milho-doce, em razão do intenso processo de seleção, apresenta pericarpo delgado e características texturais particulares do endosperma, que o fazem superior ao milho comum, quando no estado leitoso (Silva, 1994).

A fina espessura do pericarpo é uma característica das sementes de milho-doce que pode contribuir para torná-las mais suscetíveis aos danos mecânicos, e, possivelmente, aos danos térmicos durante a secagem. Wann (1986) e Wilson Junior \& Trawatha (1991) recomendam cuidados especiais nesta importante etapa da produção, que, se mal conduzida, pode comprometer a qualidade final do produto.

A relação entre o teor de umidade de equilíbrio e a umidade relativa de equilíbrio, também designada 
por atividade da água, constitui fator essencial nos projetos e estudos de sistemas de secagem, manuseio, armazenagem, embalagem e transporte e na modelagem da longevidade das sementes. Conseqüentemente, estudos têm sido feitos para obtenção de equações que expressem o teor de umidade de equilíbrio para cada produto, em razão da umidade relativa e da temperatura do ar em contato (Brooker et al., 1992; Morey et al., 1995; Chen \& Jayas, 1998). Um modelo matemático que descreva a relação entre a atividade da água (Aw), o teor de umidade e a temperatura é básico na composição dos modelos de simulação de processos na área de engenharia de processamento de produtos agrícolas. Não se encontraram, na literatura, informações a este respeito sobre sementes de milho-doce.

Diversos autores têm estudado o comportamento higroscópico de vários produtos agrícolas, utilizando métodos diferenciados para a obtenção dos dados experimentais (Sokhansanj et al., 1986; Pereira \& Queiroz, 1987; Chen \& Morey, 1989a; Mazza \& Jayas, 1990, 1991; Brooker et al., 1992; Morey et al., 1995; Chen \& Jayas, 1998; Corrêa et al., 1998a, 1998b).

Para a modelagem das curvas de equilíbrio higroscópico, têm sido utilizadas relações matemáticas semi-teóricas e empíricas, uma vez que nenhum modelo teórico desenvolvido tem sido capaz de predizer com precisão o teor de umidade de equilíbrio de grãos em todas as faixas de temperatura e umidade relativa do ar (Brooker et al., 1992).

Dentre as diversas equações utilizadas para expressar o teor de umidade de equilíbrio dos grãos em razão da temperatura e umidade relativa de equilíbrio ou atividade da água, as mais comuns, por sua relativa precisão e generalidade de uso, são as de: Henderson-Thompson, Chung-Pfost, Smith e Sabbah (Pfost et al., 1976; Sokhansanj et al., 1986; Chen \& Morey, 1989a; Brooker et al., 1992; Corrêa et al.,1995; Morey et al., 1995; Sokhansanj \& Yang, 1996; American Society of Agricultural Engineering, 1999). Entre outras equações menos conhecidas e difundidas, têm-se a de Copace (para Aw $>0,20$ ) e a de Sigma-Copace (Corrêa et al., 1995).

Chen \& Morey (1989b), trabalhando com milho amarelo, observaram que existem diferenças entre as isotermas de equilíbrio higroscópico de distintas variedades.

Este trabalho teve como objetivo determinar as curvas de dessorção de sementes de milho-doce e verificar a adequabilidade da expressão dessas curvas por modelos matemáticos.

\section{Material e Métodos}

Este trabalho foi realizado nos Departamentos de Fitotecnia e de Engenharia Agrícola da Universidade Federal de Viçosa, Viçosa, MG.

As sementes de milho-doce (Zea mays L.) das cultivares BR 400 (Superdoce) e BR 402 (Doce Cristal) foram produzidas no Município de Coimbra, MG, no ano agrícola 96/97. Após a colheita e debulha manuais, as sementes foram submetidas ao beneficiamento em máquina de ventilador e peneiras e em separador pneumático. Em seguida, foram colocadas para secagem à sombra, com a finalidade de uniformizar o teor de umidade das sementes das duas cultivares. As unidades da amostra de sementes, pesando aproximadamente $2.000 \mathrm{~g}$ e com teor de umidade médio de $17,70 \%$, em base úmida, foram acondicionadas em sacos de polietileno e armazenadas em câmara fria à temperatura aproximada de $5^{\circ} \mathrm{C}$. Antes de serem submetidas às condições de secagem até o equilíbrio higroscópico, essas sementes permaneceram à temperatura ambiente, durante 12 horas, com a finalidade de atingir o equilíbrio térmico.

As condições de temperatura e umidade relativa do ar de secagem foram obtidas e controladas por meio de uma unidade de condicionamento de ar, Aminco-Air, e monitoradas com auxílio de um psicrômetro. Os tratamentos aplicados foram as combinações das temperaturas de $30,40,50$ e $60^{\circ} \mathrm{C}$ com as umidades relativas do ar de 30, 40, 50 e $60 \%$, com fluxo de ar em torno de $25 \mathrm{~m}^{3} \mathrm{~min}^{-1} \mathrm{~m}^{-2}$. A espessura da camada de sementes foi, aproximadamente, de $2 \mathrm{~cm}$.

Durante a secagem, as unidades das amostras de sementes foram pesadas periodicamente, com a finalidade de acompanhar a perda de peso. Quando o peso não variava no limite de $2 \%$, entre três pesagens consecutivas, considerou-se que o equilíbrio higroscópico foi alcançado, sendo então determinado o teor de umidade das sementes, pelo método da estufa, a $105 \pm 3{ }^{\circ} \mathrm{C}$, por 24 horas (Brasil, 1992).

Foi adotado o delineamento experimental inteiramente casualizado, com os tratamentos dispostos em esquema 
fatorial $2 \times 4 \times 4$ ( 2 cultivares, 4 temperaturas e 4 umidades relativas), com cinco repetições.

Os seguintes modelos matemáticos foram ajustados aos dados experimentais, por análise de regressão não-linear, pelo método "Quasi-Newton", com o uso do aplicativo Statistica 5.0:

Henderson-Thompson: $\mathrm{U}_{\mathrm{e}}=[-\ln (1-\varphi) / \mathrm{a}(\mathrm{T}+\mathrm{b})]^{(1 / \mathrm{c})}$

Chung-Pfost: $\mathrm{U}_{\mathrm{e}}=\mathrm{a}-\mathrm{b} \ln [-(\mathrm{T}+\mathrm{c}) \ln \varphi]$

Smith: $U_{\mathrm{e}}=\mathrm{a}-\mathrm{bT}-\operatorname{cln}(1-\varphi)$

Sabbah: $U_{\mathrm{e}}=\mathrm{a} \varphi^{\mathrm{b}} / \mathrm{T}^{\mathrm{c}}$

Copace: $U_{e}=\exp (a-b T+c \varphi)$

Sigma-Copace: $\mathrm{U}_{\mathrm{e}}=\exp [\mathrm{a}-\mathrm{bT}+\operatorname{cexp}(\varphi)]$

onde:

$\varphi$ : umidade relativa (decimal);

$\mathrm{T}$ : temperatura ambiente $\left({ }^{\circ} \mathrm{C}\right)$;

$\mathrm{U}_{\mathrm{e}}$ : umidade de equilíbrio (\% b.s.);

a,b,c: constantes apropriadas.

A adequabilidade de cada um dos modelos para exprimir a relação da umidade de equilíbrio com a umidade relativa e a temperatura ambiente foi avaliada por meio do desvio-padrão estimado (DP). A capacidade do modelo em descrever com fidelidade o processo físico é inversamente proporcional ao valor de DP (Chen \& Morey, 1989a; Chen \& Jayas, 1998):

$\mathrm{DP}=\sqrt{\frac{\sum(\mathrm{Y}-\hat{\mathrm{Y}})^{2}}{\mathrm{GLR}}}$,

onde:

Y: valor observado experimentalmente;

$\hat{Y}$ : valor estimado pelo modelo;

GLR: graus de liberdade do resíduo.

Determinaram-se os valores da variância explicada $\left(R^{2}\right)$, expressos em porcentagem, e também foi feita a análise da distribuição dos resíduos.

\section{Resultados e Discussão}

A análise da variância da umidade de equilíbrio higroscópico mostrou efeito significativo da interação cultivar $\mathrm{x}$ temperatura $\mathrm{x}$ umidade relativa. Por essa razão, apesar da pequena diferença entre os valores médios das duas cultivares em cada combinação de temperatura e umidade relativa (Tabela 1), os modelos foram testados separadamente para cada cultivar.

Observa-se que os teores de umidade de equilíbrio são semelhantes entre as duas cultivares, embora os valores obtidos na cultivar Superdoce, em quase todos os tratamentos, sobreponham os obtidos na Doce Cristal. Ng et al. (1995) sugerem que as dife- renças de umidade de equilíbrio entre híbridos de milho são decorrentes das diferenças na composição química dos grãos.

Comparando estes resultados com os resultados referentes à umidade de equilíbrio obtidos com relação às sementes de milho comum (adaptação de American Society of Agricultural Engineering, citado por Silva, 1986) a $30^{\circ} \mathrm{C}$ e $30,40,50$ e $60 \%$ de umidade relativa, observa-se que as sementes de milho-doce apresentaram menores valores. As diferenças foram maiores na medida em que aumentava a umidade relativa do ar, alcançando valores superiores a 2\% de umidade. Quanto aos resultados obtidos com sementes de milho-pipoca (Flood Junior \& White, 1984; Corrêa et al., 1998a), também verificou-se umidade de equilíbrio inferior nas sementes de milho-doce.

A supremacia do efeito da umidade relativa do ar sobre o teor de umidade de equilíbrio, quando comparado com a temperatura, torna evidente a semelhança das duas cultivares com a maioria dos produtos agrícolas cuja higroscopicidade já foi estudada. Também de forma semelhante à maioria dos produtos higroscópicos, observa-se que os teores de umidade de equilíbrio decrescem com o aumento da temperatura e com a diminuição da umidade relativa do ar.

A apreciação dos valores do desvio-padrão e da variância explicada, bem como a distribuição dos resíduos indica que os modelos de Chung-Pfost, Sabbah e Smith foram os que melhor se ajustaram

Tabela 1. Valores médios da umidade de equilíbrio higroscópico de sementes de milho-doce, cultivares Doce Cristal e Superdoce, em diferentes combinações de temperatura e umidade relativa do ar, em base seca.

\begin{tabular}{ccccr}
\hline Temperatura $\left({ }^{\circ} \mathrm{C}\right)$ & \multicolumn{4}{c}{ Umidade relativa (\%) } \\
\cline { 2 - 5 } & 30 & 40 & 50 & 60 \\
\hline \multicolumn{5}{c}{ Doce Cristal } \\
30 & 6,73 & 8,08 & 9,22 & 10,62 \\
40 & 5,70 & 7,00 & 8,35 & 9,84 \\
50 & 5,17 & 6,28 & 7,67 & 8,98 \\
60 & 4,71 & 5,83 & 7,17 & 8,53 \\
\hline \multicolumn{5}{c}{ Superdoce } \\
30 & 7,08 & 8,27 & 9,36 & 10,72 \\
40 & 5,71 & 7,26 & 8,52 & 10,35 \\
50 & 5,12 & 6,20 & 8,06 & 9,28 \\
60 & 5,00 & 6,15 & 7,42 & 8,93 \\
\hline
\end{tabular}

Pesq. agropec. bras., Brasília, v. 36, n. 7, p. 991-995, jul. 2001 
aos dados experimentais, nas duas cultivares (Tabela 2). Os resultados do modelo de Chung-Pfost, que apresentaram uma ligeira superioridade sobre os demais (Tabela 2), podem ser observados na Figura 1. Estes resultados são inferiores aos estimados pelo mesmo modelo de Chung-Pfost com relação ao milho comum, obtido por Corrêa et al. (1995).

Tabela 2. Estimativas dos parâmetros de cada uma das equações ajustadas para estimar a umidade de equilíbrio higroscópico das sementes de milho-doce, cultivares Doce Cristal e Superdoce, com os respectivos desvios-padrão estimados (DP), variância explicada $\left(\mathrm{R}^{2}\right)$ e distribuição dos resíduos.

\begin{tabular}{|c|c|c|c|c|}
\hline Modelo & Constante & $\mathrm{DP}$ & $\mathrm{R}^{2}$ & $\begin{array}{l}\text { Distribuição } \\
\text { dos resíduos }\end{array}$ \\
\hline & \multicolumn{4}{|c|}{ Doce Cristal } \\
\hline $\begin{array}{l}\text { Henderson- } \\
\text { Thompson }\end{array}$ & $\begin{array}{l}a=4,442 \times 10^{-5} \\
b=1,776 \\
c=1,703\end{array}$ & 2,630 & 93,7 & Tendenciosa \\
\hline Chung-Pfost & $\begin{array}{l}a=25,459 \\
b=4,568 \\
c=20,533\end{array}$ & 1,124 & 98,9 & Aleatória \\
\hline Copace & $\begin{array}{l}a=1,625 \\
b=9,137 \times 10^{-3} \\
c=1,725\end{array}$ & 1,540 & 97,9 & Tendenciosa \\
\hline Sigma-Copace & $\begin{array}{l}\mathrm{a}=0,702 \\
\mathrm{~b}=9,139 \times 10^{-3} \\
\mathrm{c}=1,077\end{array}$ & 1,697 & 97,4 & Tendenciosa \\
\hline Sabbah & $\begin{array}{l}a=60,468 \\
b=0,770 \\
c=0,390\end{array}$ & 1,466 & 98,0 & Aleatória \\
\hline \multirow[t]{2}{*}{ Smith } & $\begin{array}{l}a=6,324 \\
b=-0,070 \\
c=6,978\end{array}$ & 1,380 & 98,3 & Aleatória \\
\hline & \multicolumn{4}{|c|}{ Superdoce } \\
\hline $\begin{array}{l}\text { Henderson- } \\
\text { Thompson }\end{array}$ & $\begin{array}{l}a=2,820 \times 10^{-5} \\
b=-0,757 \\
c=1,924\end{array}$ & 3,001 & 92,5 & Aleatória \\
\hline Chung-Pfost & $\begin{array}{l}a=26,877 \\
b=4,777 \\
c=25,721\end{array}$ & 1,600 & 97,8 & Aleatória \\
\hline Copace & $\begin{array}{l}\mathrm{a}=1,611 \\
\mathrm{~b}=8,447 \times 10^{-3} \\
\mathrm{c}=1,753\end{array}$ & 2,023 & 96,5 & Tendenciosa \\
\hline Sigma-Copace & $\begin{array}{l}a=0,673 \\
b=8,450 \times 10^{-3} \\
c=1,095\end{array}$ & 2,139 & 96,1 & Tendenciosa \\
\hline Sabbah & $\begin{array}{l}a=56,450 \\
b=0,781 \\
c=0,362\end{array}$ & 1,994 & 96,7 & Aleatória \\
\hline Smith & $\begin{array}{l}a=6,224 \\
b=-0,067 \\
c=7,304\end{array}$ & 1,791 & 97,3 & Aleatória \\
\hline
\end{tabular}

Pesq. agropec. bras., Brasília, v. 36, n. 7, p. 991-995, jul. 2001
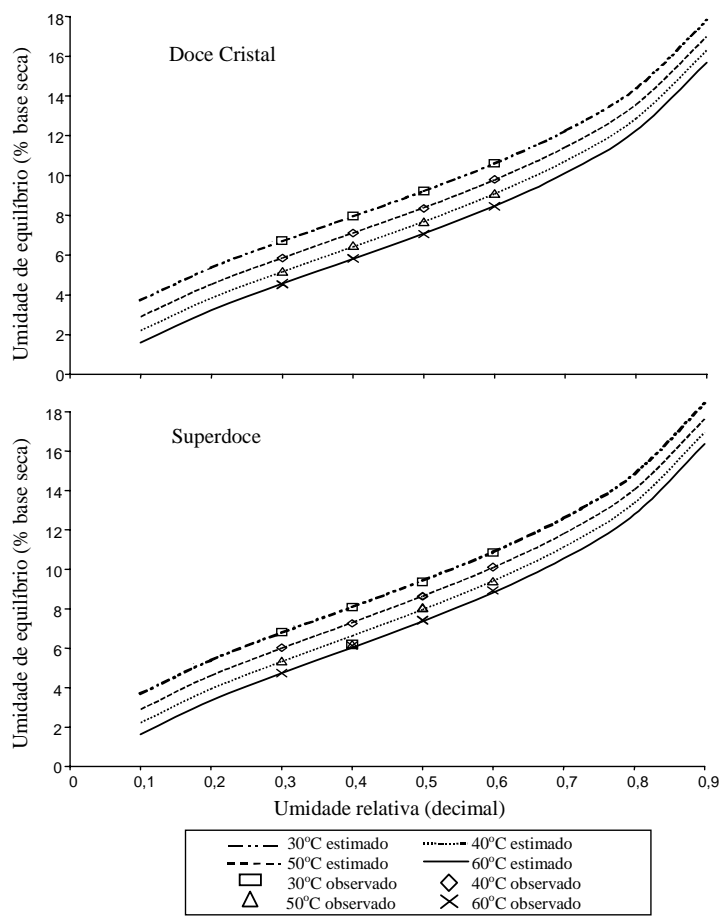

Figura 1. Curvas de umidade de equilíbrio higroscópico determinadas por valores estimados pelo modelo de Chung-Pfost e por valores observados, em sementes de milho-doce, cultivares Doce Cristal e Superdoce.

Na cultivar Superdoce, o modelo HendersonThompson foi inferior, por apresentar valores de desvio-padrão superiores e de $\mathrm{R}^{2}$ inferiores, em relação aos demais modelos (Tabela 2). Os modelos de Copace e de Sigma-Copace, em ambas as cultivares, e de Henderson-Thompson, na cultivar Doce Cristal, apresentaram distribuição de resíduos tendenciosa. Corrêa et al. (1998a) concluíram que as equações de Copace e de Sigma-Copace foram as que melhor se ajustaram aos dados experimentais das sementes de milho-pipoca; os modelos de Henderson modificado e de Chung-Pfost também foram satisfatórios.

\section{Conclusões}

1. As sementes de milho-doce das cultivares Superdoce e Doce Cristal apresentam umidades de equilíbrio higroscópico semelhantes. 
2. As equações de Chung-Pfost, Sabbah e Smith são as que melhor se ajustam aos dados, nas duas cultivares, com ligeira superioridade para a primeira.

3. O modelo de Henderson-Thompson - aplicado na cultivar Doce Cristal - e os de Copace e de Sigma-Copace - nas cultivares Doce Cristal e Superdoce - apresentam distribuição tendenciosa dos resíduos.

\section{Referências}

AMERICAN SOCIETY OF AGRICULTURAL ENGINEERING (St. Joseph, Estados Unidos). Moisture relationships of plant-based agricultural products: ASE D245.5. In: ASAE standards 1999: standards engineering practices data. 46 ed. St. Joseph, 1999. p. 512.

BRASIL. Ministério da Agricultura e Reforma Agrária. Regras para análise de sementes. Brasília, 1992. 365 p.

BROOKER, D. B.; BAKKER-ARKEMA, F. W.; HALL, C. W. Drying and storage of grains and oilseeds. Westport : AVI, 1992. $450 \mathrm{p}$.

CHEN, C.; JAYAS, D. S. Evaluation of the GAB equation for the isotherms of agricultural products. Transactions of the ASAE, St. Joseph, v. 41, n. 6, p. 1755-1760, 1998.

CHEN, C.; MOREY, V. Comparison of four EMC/ERH equations. Transactions of the ASAE, St. Joseph, v. 32, n. 3, p. $983-990,1989$ a

CHEN, C.; MOREY, V. Equilibrium relativity humidity (ERH) relationships for yellow-dent corn. Transactions of the ASAE, St. Joseph, v. 32, n. 3, p. 999-1006, 1989b.

CORRÊA, P. C.; MARTINS, D. S. R.; MELO, E. C. Umigrãos: programa para o cálculo do teor de umidade de equilíbrio para os principais produtos agrícolas. Viçosa : Centro Nacional de Treinamento em Armazenagem, 1995. $10 \mathrm{p}$.

CORRÊA, P. C.; MARTINS, J. H.; CHRIST, D.; MANTOVANI, B. H. M. Curvas de dessorção e calor latente de vaporização para as sementes de milho pipoca (Zea mays). Revista Brasileira de Engenharia Agrícola e Ambiental, Campina Grande, v. 2, n. 1, p. 75-79, 1998 a.

CORREAA, P. C.; VITAL, R. B.; MARTINS, J. H. Higroscopicidade e entalpia de vaporização para madeira de Eucalyptus grandis. Revista Árvore, Viçosa, v. 22, n. 4, p. 555-561, 1998b.

FLOOD JUNIOR, C. A.; WHITE, G. M. Desorption equilibrium moisture relationships for popcorn. Transactions of the ASAE, St. Joseph, v. 27, n. 3, p. 561571,1984
MAZZA, G.; JAYAS, D. S. Equilibrium moisture characteristics of sunflower seeds, hulls, and kernels. Transactions of the ASAE, St. Joseph, v. 34, n. 2, p. 534538, 1991.

MAZZA, G.; JAYAS, D. S.; WHITE, N. D. G. Moisture sorption isotherms of flax seed. Transactions of the ASAE, St. Joseph, v. 33, n. 4, p. 1313-1318, 1990.

MOREY, V.; WILCKE, W. F.; MERONUCK, R. A.; LANG, J. P. Relationship between equilibrium relative humidity and deterioration of shelled corn. Transactions of the ASAE, St. Joseph, v. 38, n. 4, p. 1139-1145, 1995.

NG, H. F.; MOREY, R. V.; WILCKE, W. F.; MERONUCK, R. A.; LANG, J. P. Relationship between equilibrium relative humidity and deterioration of shelled corn. Transactions of the ASAE, St. Joseph, v. 38, n. 4, p. 11391145, 1995.

PEREIRA, J. A. M.; QUEIROZ, D. M. de. Higroscopia. Viçosa : Centro Nacional de Treinamento em Armazenagem, 1987. $28 \mathrm{p}$

PFOST, H. B.; MAURER, S. G.; CHUNG, D. S.; MILLIKEN, G. Summarizing and reporting equilibrium moisture data for grains. St. Joseph : ASAE, 1976. 11 p. (Paper, 76-3520).

SILVA, N. Melhoramento de milho doce. In: ENCONTRO SOBRE TEMAS DE GENÉTICA E MELHORAMENTO, 11., 1994, Piracicaba. Anais... Piracicaba : ESALQ, 1994. v. 11, p. 45-49.

SILVA, W. R. Secagem das sementes. In: CÍCERO, S. M.; MARCOS FILHO, J.; SILVA, W. R. (Org.). Atualização em produção de sementes. Campinas : Fundação Cargill, 1986. p. 155-182.

SOKHANSANJ, S.; YANG, W. Revision of the ASAE standard D245.4: moisture relationships of grains. Transactions of the ASAE, St. Joseph, v. 39, n. 2, p. 639642, 1996.

SOKHANSANJ, S.; ZHIJIE, W.; JAYAS, D. S.; KAMEOKA, T. Equilibrium relative humidity- moisture content of rapeseed (canola) from $5^{\circ} \mathrm{C}$ to $25^{\circ} \mathrm{C}$. Transactions of the ASAE, St. Joseph, v. 29, n. 3, p. 837839, 1986.

WANN, E. V. Leaching of metabolites during imbibition of sweet corn seed of different endosperm genotypes. Crop Science, Madison, v. 26, p. 731-733, 1986.

WILSON JUNIOR, D. O.; TRAWATHA, S. E Physiological maturity and vigor in production of 'Florida Staysweet' Shrunken-2 sweet corn. Crop Science, Madison, v. 31, p. 1640-1647, 1991. 